\title{
EDITORIAL
}

\section{Personalised medicine for cystic fibrosis: treating the basic defect}

\author{
J. Stuart Elborn
}

\section{THE RIGHT TREATMENT, FOR THE RIGHT PATIENT, AT THE RIGHT TIME}

The concept of personalised or stratified therapies is not a new one. For centuries, physicians have observed that the manifestation of a disease and its response to intervention can vary according to many factors including age, sex, ethnicity, diet and the type of administered drug [1]. Hippocrates, for example, observed over 2,000 years ago that patients can respond very differently to various medications [2].

It wasn't until 1998, however, that the term "personalised therapy" was first used [3]. This can be defined as a therapy prescribed using molecular profiling technologies to tailor the right therapeutic strategy for the right person at the right time. The therapy is typically accompanied by a "companion test" or clinical biomarker to identify responders whilst assessing therapeutic response.

Personalised medicine is often used synonymously with the expression "stratified medicine". There are, however, subtle differences between both of these terms as illustrated by the patient therapeutic continuum described by TRUSHEIM et al. (fig. 1) [4].

Most of today's medical practice is empirical, with drug development targeted at treating large populations of patients; an example is non-steroidal anti-inflammatory drugs, which have high efficacy in almost the entire population (fig. 1).

Other agents, however, may have a significant number of nonresponders, as is the case with selective serotonin-reuptake inhibitors for the treatment of depression. Identifying and specifically treating this subpopulation of patients is stratified medicine [5]. A well-known example is the monoclonal antibody trastuzumab, which represents one of the first targeted adjunctive therapies for the treatment of breast cancer in patients who overexpress HER2/neu, the gene for human epidermal growth factor receptor 2 [6].

The most specific level in the patient therapeutic continuum of care is personalised or individualised medicine. An extreme

Centre for Infection and Immunity, School of Medicine, Dentistry and Biomedical Sciences, Queens University, Belfast, UK

CORRESPONDENCE: J.S. Elborn, Centre for Infection and Immunity, School of Medicine, Dentistry and Biomedical Sciences, Queens University, Health Sciences Building, 97 Lisburn Road, Belfast, BT9 7BL, UK. E-mail: s.elborn@qub.ac.uk

PROVENANCE: Publication of this peer-reviewed article was supported by Vertex Pharmaceuticals Inc., USA (principal sponsor, European Respiratory Review issue 127). example of this is development of a vaccine for an individual patient. Oncophage and provenge are both examples of therapeutic "cancer vaccines" for the treatment of renal cell carcinoma and prostate cancer, respectively [7]. Both therapies are tailor made to raise a specific immune response against the molecular profile of the patient's cancer.

\section{PERSONALISED MEDICINE AND RESPIRATORY DISEASE}

Respiratory medicine is at the forefront of personalised medicine, as exemplified by recent advances in asthma research. Asthma, as a complex multifactorial disease, poses a significant challenge for genomics-led drug discovery. The disease pathophysiology involves the interaction of multiple genes with each other, and with a plethora of environmental factors, such as allergens, pathogens and air pollution [8]. Nevertheless, over the past few years there has been significant progress in identifying asthma-associated genes, primarily driven by genome-wide association studies [9, 10]. Gene expression profiling will not only allow accurate profiling of responders and non-responders to particular therapies, but will also aid identification of novel therapeutic targets for drug discovery. Ultimately, this could lead to asthma management plans tailored to individual patients.
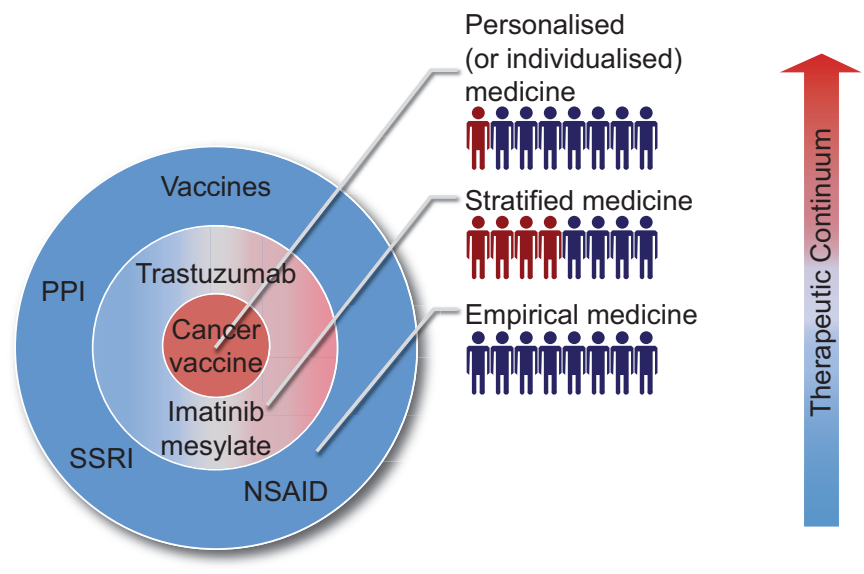

FIGURE 1. The spectrum of therapeutic care: from empirical medicine to personalised medicine. PPI: proton pump inhibitor; SSRI: serotonin reuptake inhibitor; NSAID: non-steroidal anti-inflammatory drug. 


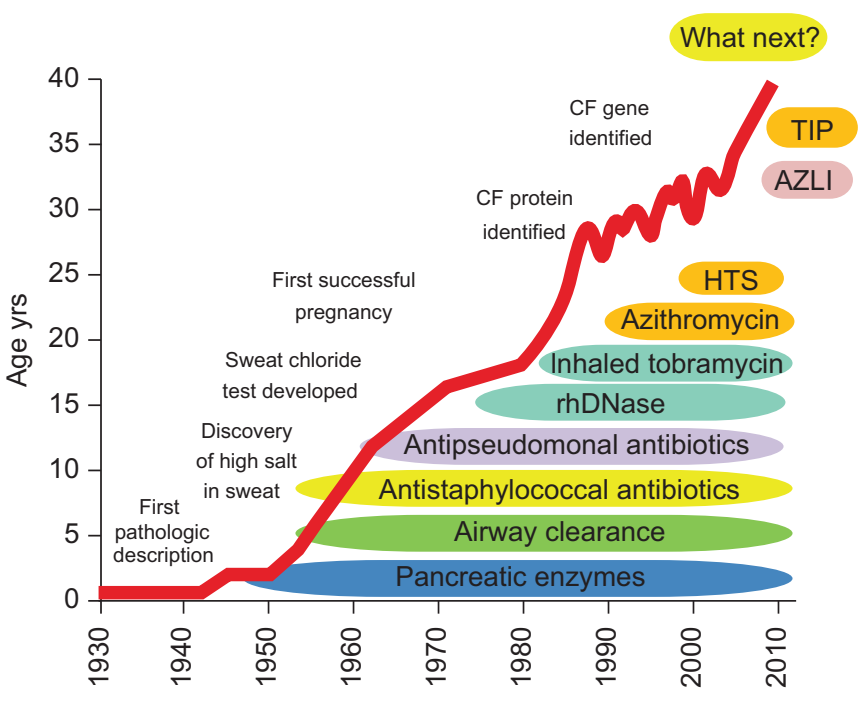

FIGURE 2. Schematic illustration of how the introduction of novel cystic fibrosis (CF) therapies influenced patient survival over the decades. HTS: high throughput screening; AZLI: aztreonam for inhalation solution; TIP: tobramycin inhalation solution.

\section{CYSTIC FIBROSIS AS A PARADIGM FOR PROSPECTIVE PERSONALISED DRUG DEVELOPMENT}

Cystic fibrosis (CF) is an inherited disease affecting the respiratory, digestive and reproductive systems [11]. The disease is caused by mutations in the cystic fibrosis transmembrane conductance regulator (CFTR) gene, colloquially referred to as the CF gene. When CF was first described in 1938, the predicted survival age of patients was only 6 months [12]. Over the decades the prognosis has incrementally increased due to the introduction of multiple therapies that treat the symptoms of CF (fig. 2). Despite these improvements, however, the predicted survival age of CF patients was reported as only 37 yrs as recently as 2009 [12].

$\mathrm{CF}$ as a disease area is well positioned to take advantage of personalised medicine. It is a monogenic disorder (i.e. is the result of mutation(s) in a specific gene) and has a well-characterised pathophysiology with clear therapeutic targets [13]. Furthermore, diagnosis of $\mathrm{CF}$ often utilises genetic testing, leading to a high rate of mutation identification in the CF population [14]. Research into CFTR gene mutations continues to reveal correlations between various CFTR genotypes and disease severity [15].

Recently, a first-in-class disease-modifying CF treatment was approved in the USA and Europe for patients with a specific mutation in their CFTR gene. The clinical development of this small molecule CFTR modulator (ivacaftor) serves as a promising example of how personalised therapies can transform the therapeutic landscape.

This series of articles has been developed based on the symposium "Personalized medicine: Treatment advances and the genetic medical revolution", which was held during the 2012 European Respiratory Society Congress in Vienna, Austria. The articles in this series will discuss the potential impact of personalised medicine approaches on respiratory medicine in light of the recent development of CFTR modulators.
HALL [16] will begin by introducing the concepts of personalised medicine and how this approach differs from traditional treatment management approaches, and provide a number of examples in the field of oncology before examining recent salient findings in respiratory medicine. DeRICHS [17] will provide an overview of the genetics and pathophysiology of $\mathrm{CF}$ and how this knowledge is being applied to CF drug discovery. He will also comment on the various classes of CFTR mutations and how they can be targeted by CFTR modulators, as well as briefly examining the proposed mechanisms of action of CFTR modulators before focusing on the pre-clinical development of ivacaftor. SERMET-GAUDELUS [18] will explore the clinical translation of CFTR modulators by reviewing data from ivacaftor clinical trials in CF patients with a specific CFTR mutation called G551D, and conclude by commenting on the potential future impact of CFTR modulators on $\mathrm{CF}$ care.

The prospects of developing personalised therapies in the field of respiratory medicine look promising, as illustrated by the ivacaftor clinical development programme in CF. Nevertheless, the challenges faced by researchers developing personalised therapies remain significant, especially in more complex polygenetic diseases, such as asthma. Overcoming these challenges will require collaboration between public bodies, such as academic institutions, regulatory authorities and clinical networks, and commercial organisations, such as pharmaceutical and diagnostic companies [19].

\section{STATEMENT OF INTEREST}

J.S. Elborn has received fees for consultancy and speaking from Vertex. He received payment as a grant for three clinical trials $(€ 50,000)$.

\section{REFERENCES}

1 Jain KK. Personalized medicine. Curr Opin Mol Ther 2002; 4: 548-558.

2 Sykiotis GP, Kalliolias GD, Papavassiliou AG. Pharmacogenetic principles in the Hippocratic writings. J Clin Pharmacol 2005; 45: $1218-1220$.

3 Jain KK, ed. Textbook of Personalised Medicine. New York, Springer, 2009.

4 Trusheim MR, Berndt ER, Douglas FL. Stratified medicine: strategic and economic implications of combining drugs and clinical biomarkers. Nat Rev Drug Discov 2007; 6: 287-293.

5 Ginsburg GS, Willard HF. Genomic and personalized medicine: foundations and applications. Transl Res 2009; 154: 277-287.

6 Olopade OI, Grushko TA, Nanda R, et al. Advances in breast cancer: pathways to personalized medicine. Clin Cancer Res 2008; 14: 7988-7999.

7 Camarero J, Ruiz S. Cancer immunotherapy products: regulatory aspects in the European Union. Hum Vaccin Immunother 2012; 8: 1354-1359.

8 Tse SM, Tantisira K, Weiss ST. The pharmacogenetics and pharmacogenomics of asthma therapy. Pharmacogenomics J 2011; 11: 383-392.

9 Portelli M, Sayers I. Genetic basis for personalized medicine in asthma. Expert Rev Respir Med 2012; 6: 223-236.

10 Hall IP, Sayers I. Pharmacogenetics and asthma: false hope or new dawn? Eur Respir J 2007; 29: 1239-1245.

11 Rowe SM, Miller S, Sorscher EJ. Cystic fibrosis. N Engl J Med 2005; 352: 1992-2001.

12 Cohen-Cymberknoh M, Shoseyov D, Kerem E. Managing cystic fibrosis: strategies that increase life expectancy and improve quality of life. Am J Respir Crit Care Med 2011; 183: 1463-1471. 
13 Amaral MD. Targeting CFTR: how to treat cystic fibrosis by CFTRrepairing therapies. Curr Drug Targets 2011; 12: 683-693.

14 De Boeck K, Derichs N, Fajac I, et al. New clinical diagnostic procedures for cystic fibrosis in Europe. J Cyst Fibros 2011; 10: Suppl. 2, S53-S66.

15 McKone EF, Goss CH, Aitken ML. CFTR genotype as a predictor of prognosis in cystic fibrosis. Chest 2006; 130: 1441-1447.

16 Hall IP. Stratified medicine: drugs meet genetics. Eur Respir Rev 2013; 22: 53-57.
17 Derichs N. Targeting a genetic defect: cystic fibrosis transmembrane conductance regulator modulators in cystic fibrosis. Eur Respir Rev 2013; 22: 58-65.

18 Sermet-Gaudelus I. Ivacaftor treatment in patients with cystic fibrosis and the G551D-CFTR mutation. Eur Respir Rev 2013; 22: $66-71$.

19 Ginsburg GS, McCarthy JJ. Personalized medicine: revolutionizing drug discovery and patient care. Trends Biotechnol 2001; 19: 491-496. 